I enjoyed reading the book, although I was irritated by the breathless, adulatory style and by the use of English so American that the old joke about 'two cultures divided by a common language' surfaces yet again. I also have reservations about the scientific value of the book for the reasons given below, but I must emphasize that the scientific data presented are both accurate and comprehensive.

Snow's experiences in Argentina are worthy of record, and it is right that the memories of this period of oppression should be preserved. Although Witness from the Grave has been widely researched, the references are of limited value. Many are from press-cuttings libraries, or from material that is unpublished or not 'readily available', or from personal communications. There are, unfortunately few citations of

\section{A symbiotic relationship}

Demosthenes Kazanas

Cosmlc Rays and Particle Physics. By Thomas K. Gaisser. Cambridge University Press: 1991. Pp. 279. Hbk £35, \$65; pbk $£ 15, \$ 24.95$.

THERE can hardly be anyone in physical sciences who has not become aware of the interplay between the two diverse disciplines of particle physics and cosmology that has stormed through both scientific and popular editions in the decade past. Although the impact of the developments in particle physics on cosmology (and the other way around to a lesser degree) has no doubt been great, this interplay has been mainly confined to the purely theoretical domain (with the exception of the limits on the number of neutrino flavours set by Big Bang nucleosynthesis and confirmed by LEP at CERN). But the hard facts have been less forthcoming than one would have guessed from the flurry of theoretical model-dependent pronouncements. Although this is the case for the interplay between cosmology and particle

\section{New in paperback}

- James Joule: A Biography by Donald S. L. Cardwell published by Manchester University Press/St Martin's Press at £12.95, $\$ 24.95$. - Wonderful Life: The Burgess Shale and the Nature of History by Stephen Gould published by Penguin/Norton at $£ 6.99, \$ 10.95$.

- The Deluge and the Ark: A Journey into Primate Worlds by Dale Peterson published by Avon at \$11.95.

- Genetically Engineered Organisms: Benefits and Risks by J. R. S. Fincham and J. R. Ravetz published by the Open University Press at £8.99.

- Liquid Crystals: Nature's Delicate Phase of Matter by Peter J. Collings published by Princeton University Press/Adam Hilger at $\$ 10.95$, £9.95. major texts and papers.

I am unable to recommend this book to the serious scientist or academic department. The information is better obtained from the works of authors such as W. M. Krogman and K. J. Reichs, or from the mainstream journals. Witness will, however, be of use to lay readers with an interest in criminalistics, although they might jib at the price. Some lawyers or police officers may also find it interesting.

I hope the book does well, but unfortunately it falls between two stools; it is too general for the specialist and too wordy and expensive to have more than a limited public appeal.

M. A. Green is at the Medico-legal Centre, Watery Street, Sheffield S3 7ES, UK.

physics, a similar symbiotic relation between the disciplines of particle and cosmic-ray physics has been growing quietly with a lot more relevance to experimental facts, and it is the subject of Cosmic Rays and Particle Physics by Thomas Gaisser.

Cosmic rays have been the subject of intense scientific study during the past several decades, and it is worth remembering that several of the discoveries in particle physics were made using the cosmic-ray beam. But the advent of laboratory accelerators has led progressively to the decline in significance of the cosmic-ray beam as a means for studying high-energy physics. The emphasis in cosmic-ray studies subsequently shifted to more astrophysically oriented issues, such as the spectrum, composition, and acceleration of cosmic rays, whose resolution, incidentally, is likely to remain elusive for the foreseeable future. So at the end of the last decade, the field of cosmic-ray physics appeared mature enough to preclude the surprises and quick advances reserved for subjects of a more youthful age.

At this point the latest developments in particle physics have forced upon us several facts that have had an impact on the study of cosmic-ray physics. For example, the discovery of new quark flavours with very short life-times has suggested the presence of a prompt muon component in the atmospheric cosmic-ray cascades (that is, muons emitted from meson decay before the parent meson had suffered any collisions in the atmosphere). More importantly, the search for proton decay, motivated by theoretical considerations, has prompted the construction of a number of large underground detectors for which the atmospheric muon and neutrino-induced events produced by the cosmic radiation beam constitute the background rather than the signal. Cosmic-ray physicists have thus been forced to refine their calculations to determine this background as best as possible. To this goal they have received help from accelerator data, which can now provide information about cross sections up to $\sim 1 \mathrm{TeV}$ at the centre of mass, thus further reinforcing the symbiotic relation between high-energy and cosmicray physics and providing an unexpected boost to the latter. It is precisely the analysis of the physics of the cosmic-ray-induced high-energy cascades and the details associated with the detection of the various components (for example, electrons, muons, neutrinos) both on and under ground that constitutes the main thrust of this book. In this respect, the material covered in the book does not contain as much novelty of notions and ideas as one might expect having been exposed to the interplay between particle physics and cosmology. But it contains a lot of material and facts that are relevant to reallife detectors and observations as they have been shaped by the latest advances in highenergy physics and astrophysical discoveries. Discussions of some more speculative, exotic stuff, such as neutrino oscillations, are included, but then only within the context of their impact on observations with and on limits set by real detectors.

The apparent absence of any significant proton decay and the nearly simultaneous discovery of point sources of very high $\left(10^{12}\right.$ $\mathrm{eV})$ and ultra-high $\left(10^{15} \mathrm{eV}\right)$ radiation associated with compact accreting objects in our Galaxy, endowed the proton-decay detectors with the alternative function of high-energy astrophysics telescopes. This additional function did in fact cause quite a stir with the tentative report of muon-rich showers (that is, richer than expected from photon-initiated cascades) in these detectors from the direction of one of these astrophysical ultra-high-energy sources, hinting at the possibility of new physics at $\mathrm{TeV}$ energies. The major theoretical and experimental issues associated with these sources are also reviewed in the book. In addition, one can find a succinct but lucid exposition of a variety of other topics related to cosmic-ray research, such as shock acceleration, propagation, the role of antiprotons as probes of cosmic-ray sources and propagation and so on.

Cosmic Rays and Particle Physics offers quite a thorough overview of the physics of cosmic-ray cascades in the atmosphere and underground as they have been shaped by the needs and developments of high-energy physics in the last decade. The treatment of all the topics is concise and based on or supplemented by sufficient physical argumentation to allow a much clearer understanding of the accompanying equations. The presentation is excellent and very useful to graduate students (to whom the book is mainly addressed) and also for researchers wishing to familiarize themselves with cosmic-ray cascades and cosmic rays in general, and there are a number of reviews and references provided that should suffice as a first step for a more thorough study.

Demosthenes Kazanas is at the NASA/Goddard Space Flight Center, Greenbelt, Maryland 20771, USA. 